\title{
AORTIC STENT GRAFTING AND SIDE-BRANCH EMBOLIZATION IN AN EXPANDING CHRONIC TYPE B DISSECTION
}

Bertrand Janne d'Othée, MDa

Hervé Rousseau, MD ${ }^{\mathrm{a}}$

Philippe Soula, MD $^{\mathrm{b}}$

Bruno Dongay, $\mathrm{MD}^{\mathrm{c}}$

Maria Ines Millan, MDa

Michel Galinier, $\mathrm{MD}^{\mathrm{c}}$

Pierre Massabuau, $\mathrm{MD}^{\mathrm{c}}$

Francis Joffre, MD

Philippe Otal, $\mathrm{MD}^{\mathrm{a}}$
Objective: This is a report of endovascular treatment of a case of type $B$ thoracoabdominal aortic dissection in a patient with progressive dyspnea, dorsolumbar pain, and expanding aortic diameter over a 1-year period. Methods: Pretreatment imaging evaluation showed that the false lumen supplied only the celiac trunk. Endovascular treatment combined (1) embolization of the first segment of the celiac trunk to avoid distal back-flow into the false lumen and (2) stent grafting to occlude the initial entry tear. Results: The treatment resulted in technical and clinical success. The patient remains asymptomatic 12 months after treatment. Conclusion: Stent grafting offers an interesting therapeutic alternative to exclude the initial entry tear in aortic dissection and may be combined with other endovascular procedures. (J Thorac Cardiovasc Surg 1999; 118:1021-5)
$S^{t+o n g}$ tent grafting of the thoracic aorta has emerged as a new technique in the treatment of aneurysms of various causes and traumatic ruptures of the aortic isthmus. This case report illustrates a different indication for this promising technique. We report a case of type B thoracoabdominal aortic dissection managed by a combination of stent grafting for exclusion of the tear and embolization to avoid distal back-flow.

\section{Clinical summary}

A 62-year-old man with severe thoracic pain was given a diagnosis of a Stanford type B dissection of the thoracoabdominal aorta and was successfully managed by using conservative medical therapy. He had a previous history of heavy smoking and had been taking antihypertensive medication for the past 35 years. Transesophageal echography (TEE), computed tomography (CT), and magnetic resonance imaging showed one large primary entry site at the supradiaphragmatic level (Fig 1, right), and both retrograde and antegrade extensions were noted. The false lumen was partially thrombosed in its upper portion, whereas its circulating portion supplied the celiac trunk only (Fig 1, left). The myointimal flap

From the Department of Radiology, ${ }^{\text {a }}$ the Department of Cardiovascular Surgery, ${ }^{\mathrm{b}}$ and the Department of Cardiology, ${ }^{\mathrm{c}}$ Centre Hospitalier Universitaire, Hôpital de Rangueil, Toulouse, France.

Received for publication Nov 24, 1998; revisions requested Jan 19, 1999; revisions received June 22, 1999; accepted for publication Aug 11, 1999.

Address for reprints: Professor H. Rousseau, MD, Service de Radiologie, C.H.U. Rangueil, 1, Avenue Jean Poulhès, 31403 Toulouse Cedex 4, France.

Copyright (C) 1999 by Mosby, Inc.

$0022-5223 / 99 \$ 8.00+0 \quad \mathbf{1 2} / \mathbf{1} / \mathbf{1 0 2 0 8 6}$ was thick and barely mobile. Both renal arteries and the superior mesenteric artery were patent and fully supplied by the true lumen. Maximal diameter of the descending aorta was estimated at $33 \mathrm{~mm}$.

Follow-up over a period of 12 months revealed the development of a pseudoaneurysmal dilation of the false lumen, which showed partial thrombosis. Maximum diameter of the descending aorta was $59 \mathrm{~mm}$. Furthermore, because the patient had dorsolumbar pain and increasing dyspnea over a 10-month period, an endovascular procedure was performed after achieving general anesthesia by a combined radiologic and surgical team. The primary entry tear was closed with a covered stent to stop aortic expansion and to avoid subsequent rupture. Aortography showed that intercostal arteries arose from the true lumen only. As a result of the anatomic presentation of the false lumen, it was believed that possible backflow reinjection of this channel could occur from the celiac trunk. After a radiograph of the superior mesenteric artery confirmed the patency of collaterals to the liver through the gastroduodenal artery, an embolization of the first proximal centimeters of the celiac trunk was performed through the false lumen before stent-graft placement by using detachable balloons and metallic coils. This false lumen catheterization was only possible from a right brachial approach through the primary entry tear. Complete occlusion of the first segment of the celiac trunk was proved by angiography before stent grafting the thoracic aorta during the same session.

Stent-graft placement was performed under TEE and angiographic guidance on the entry tear by using a Talent stent graft (World Medical Manufacturing Corp, Sunrise, Fla) of $100 \mathrm{~mm}$ in length and $35 \mathrm{~mm}$ in deployed diameter. This device was bare on its first $15 \mathrm{~mm}$. Because of the 24F caliber of the introducer, a right common iliac arteriotomy was necessary to allow introduction of the undeployed stent graft. The covered portion ( $85 \mathrm{~mm}$ long) was implanted just 


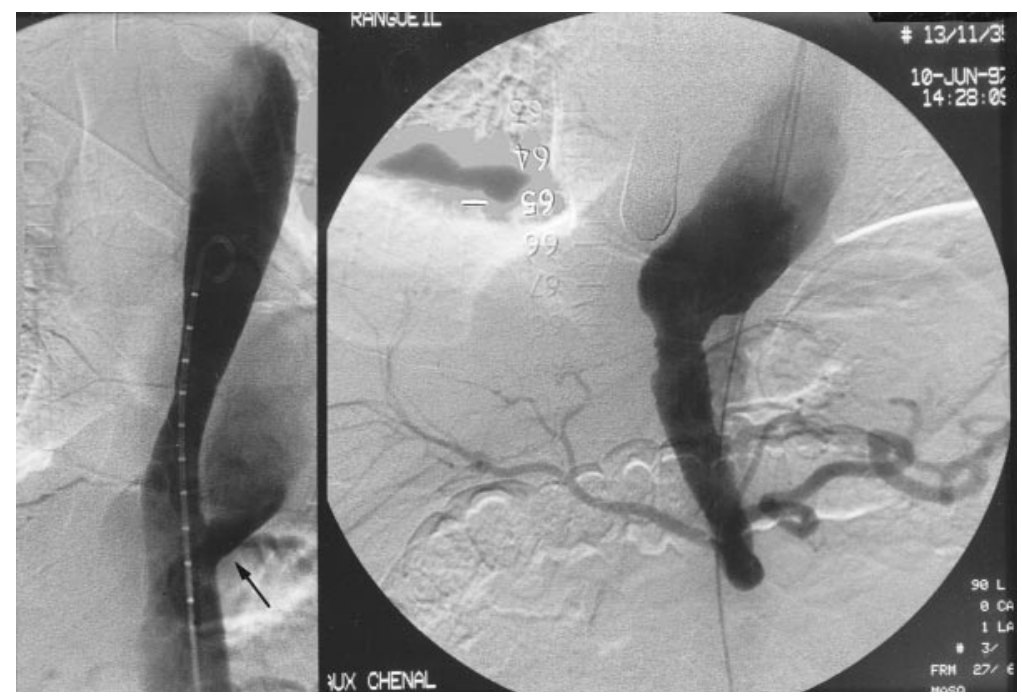

Fig 1. Digital subtraction arteriography with a calibrated pigtail catheter performed before endovascular procedure. Left, Injection in the true lumen at the supradiaphragmatic level shows the initial entry site (arrow) and retrograde opacification of the false lumen up to the aortic isthmus. Antegrade extension up to the L1 level is noted. Right, Digital subtraction arteriography (brachial approach). Injection in the false lumen is followed by opacification of the celiac trunk and its branches.

above the level of the aortic diaphragmatic orifice to obliterate the entry tear.

End-procedure arteriographic (Fig 2) and TEE studies showed immediate exclusion of the false lumen and a slightly irregular inner surface of the stent graft. A retrograde opacification of the distal branches of the celiac trunk (true lumen) from the pancreaticoduodenal arteries was observed without retrograde opacification of the embolized proximal false lumen. No paraplegia was observed, and the pain decreased.

A few days later, a subacute ischemia of the right upper limb developed, which required desobstruction by using a Fogarty probe. The patient had an immediate recovery of his peripheral pulses. He remained asymptomatic for the next 12 months, with a spiral CT showing a maximal aortic diameter of $50 \mathrm{~mm}$ (Figs 3 and 4).

\section{Discussion}

Recent advances in the pathophysiology of aortic dissections have enabled different kinds of endovascular treatment for complicated acute and chronic dissections involving the descending thoracic and abdominal aorta. The therapeutic goals are (1) to avoid ischemia caused by obstruction by malpositioning of the myointimal flap or (2) to prevent pseudoaneurysmal dilation of the false lumen and potential rupture.

In obstructions, several techniques have been advocated to relieve the pressure gradient between the true and false lumina, such as fenestration and bare stenting of the aorta or of the compromized aortic branches.
Stent grafting offers an interesting alternative to occlude the primary entry tear and the residual false lumen at the level of the descending aorta to avoid aortic dilation and rupture. However, covering of the main entry site is technically more difficult in dissections than in aortic dilations of other causes, such as degenerative aneurysms or traumatic pseudoaneurysms. Actually, the particular spatial configuration of aortic dissections implies asymmetric channels that appear noncircular on cross-sectional views. The anchoring of the stent graft to the wall is imperfect in those conditions, facilitating leakage between the device and the aortic wall. ${ }^{1}$ Furthermore, obliteration of the false lumen may be particularly difficult in chronic dissections in which the contracted myointimal flap has become fibrotic and thickened, impeding correct deployment of the stent graft despite appropriate radial force.

Prevention of rupture by stent grafting involves 2 distinct mechanisms: compression and obliteration of the false lumen in case of small primary entry tears and relatively low false lumen flow or induction of false lumen thrombosis by isolating it from the circulating true lumen. ${ }^{1}$ Controversies ${ }^{1,2}$ remain regarding the material and technique needed for endovascular closure of the primary entry site. At first, some authors tried to complete this closure by inflating balloon catheters and then using noncovered stents. Varied types of metallic stents were used experimentally to 
reopen the true lumen, to restore distal flow through the true lumen, and to close the false lumen. ${ }^{1,3-5}$ This last objective could be fully achieved only with extensive bare stenting of the true lumen: noncovered stents placed only on the proximal portion of experimental aortic dissections induced false lumen thrombosis at the same level but could not prevent persisting patency of the distal false lumen from reentry orifices, ${ }^{1,5}$ even when intraoperative or postoperative anticoagulation was avoided. However, the true significance of this persisting patency of the false lumen needs further studies to determine its true potential gravity. Indeed, persistence of a circulating false lumen is a well-known and frequent $(85 \%)$ feature observed after an otherwise successful surgical repair, and it warrants serial imaging follow-up. It is feared, but not proved, that it could promote false aneurysm formation over the long term. On the other hand, it may be the only route of blood flow to major organs in some patients. ${ }^{1}$

Whether one should cover either the primary entry tear only or the whole false channel with the stent graft remains unclear. In the first hypothesis 2 Japanese studies with experimental stent grafts, either with polyurethane embedding ${ }^{2}$ or covering, ${ }^{3}$ showed complete closure of the false lumen, whereas another study showed persistent reentry and absence of total false lumen thrombosis. 5

The need to embolize aortic side branches to avoid back-flow and retrograde leak under the stent graft is obviously of main concern. This choice implies a risk of distal ischemia. Embolization of side branches of the aorta has been reported as an adjunct endovascular technique in stent grafting of abdominal aortic aneurysms to thrombose perigraft leaks and to stop back-bleeding under the stent graft originating from retrograde injection of collateral arteries. A different purpose was followed in our case: we wanted to prevent retrograde filling from the celiac trunk false lumen to the aortic false lumen, even if the celiac trunk ostium was located well below the distal end of the stent graft. We therefore needed to block only the proximal part of the celiac trunk, like a surgical ligation, without producing distal ischemia. Long-term experience is now available with proximal transcatheter embolization of the visceral trunks. It has been shown that blocking of the common hepatic artery was not associated with liver ischemia so long as patency of both the arterial collaterals (mainly patency of the gastroduodenal artery and its branches) and the portal vein was ensured. In our patient this last point was ensured by the arteriographic study performed before stent grafting. Furthermore, embolization had to be achieved

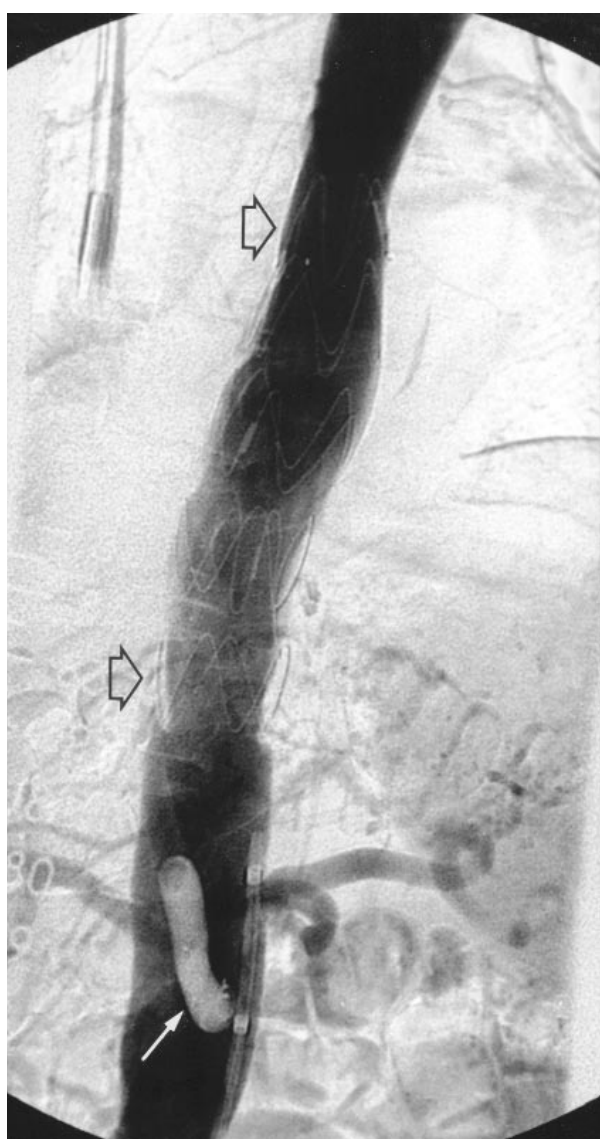

Fig 2. Follow-up arteriogram at the end of the endovascular therapeutic procedure. Occlusive balloons (white arrows) and coils have been placed in the proximal portion of the celiac trunk. Stent graft (black arrows) is seen above this level. The false channel is no longer opacified.

before stent graft deployment because the Dacron covering would have blocked any later endovascular access to the celiac trunk through the false lumen.

Because the false lumen fully supplied the celiac trunk, combined stent grafting and proximal celiac trunk embolization led to complete false lumen thrombosis. On the other hand, we have also treated several patients with distal reentries by means of the endovascular approach. Those resulted in local thrombosis of the false lumen at the level of the stent graft, although the distal portion of the false lumen remained patent at the level of the reentries. No retrograde endoleak through the false lumen was observed under the stent graft in any case.

Although first used as an alternative therapy in patients with inoperable disease, stent grafting offers an interesting alternative in dissections and several other critical diseases involving the descending tho- 

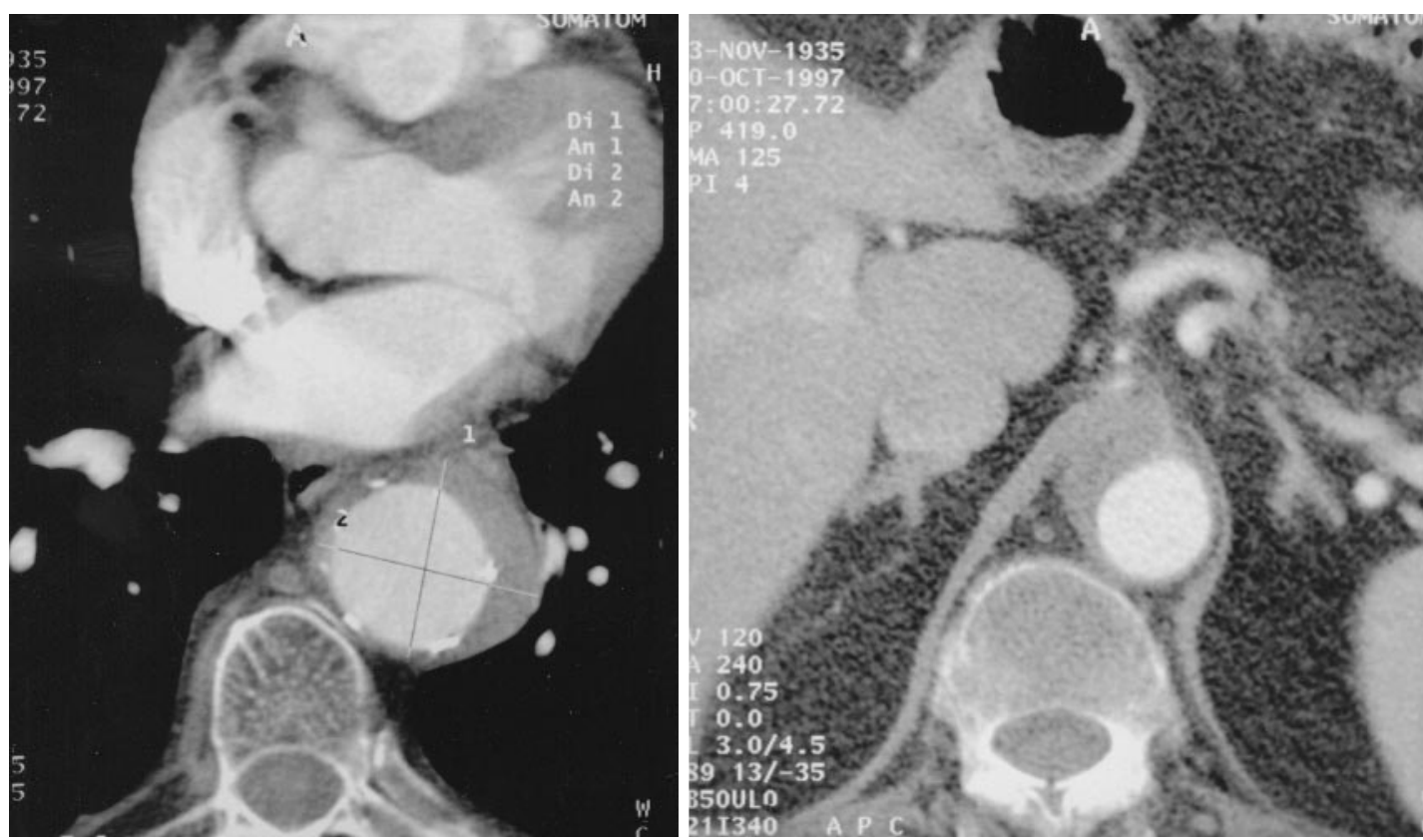

Fig 3. Axial CT slice 4 months after endovascular treatment. The false channel has thrombosed. Branches of the celiac trunk are opacified.

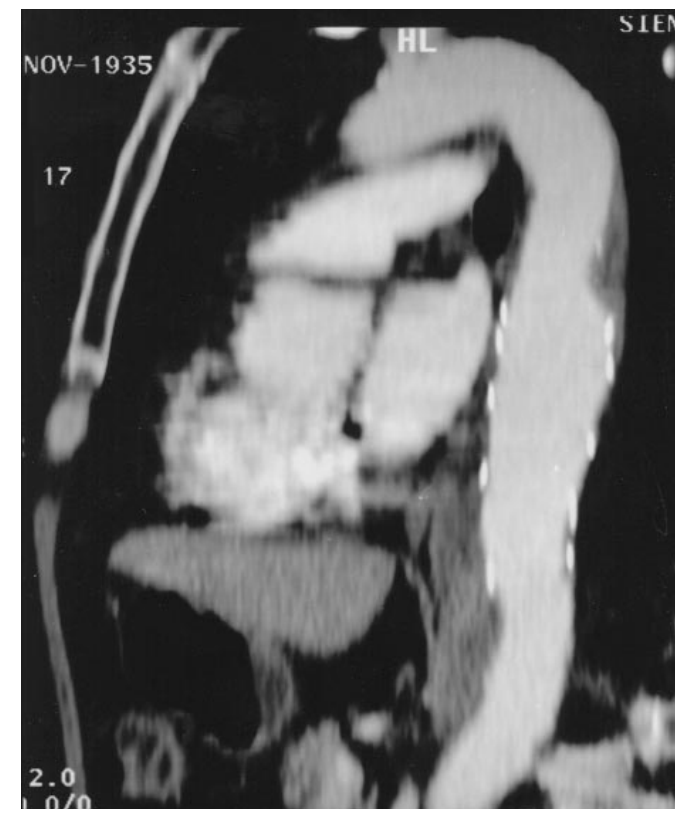

Fig 4. Maximum intensity projection reconstructed from spiral CT acquisition 12 months after endovascular treatment. The stent graft stays patent.

racic aorta. These patients often have a precarious cardiorespiratory status and sometimes have a high operative risk. Even if stent grafting has been performed after achievement of general anesthesia to date, it does not need extracorporeal circulation and requires only a slight heparinization and moderate and transient hypotension during deployment. Furthermore, previous thoracotomies could promote mediastinal fibrosis, which enhances difficulties in surgical dissection of the thoracic aorta, whereas this fibrosis does not interfere with stent graft placement. And even if stent graft manufacturing still requires several weeks before completion, future improvements could probably allow storage of these devices in variable sizes in the endovascular suite, making possible immediate treatment at the time of initial diagnostic angiography. Although these initial results are encouraging, further studies with larger follow-up are necessary.

\section{Conclusion}

We believe that the varied manifestations of complicated aortic dissections may benefit from numerous emerging endovascular techniques. Good understanding of the pathophysiology is the key element to select indications among those various treatments. Further long-term and experimental studies are needed to extend their clinical field of application.

We thank Miss Marie-Laure Vidal for her assistance with the manuscript. 


\section{REFERENCES}

1. Kato N, Hirano T, Takeda K, Nakagawa T, Mizumoto T, Yuasa $\mathrm{H}$. Treatment of acute aortic dissections with expandable metallic stents: experimental study. J Vasc Interv Radiol 1994;5:41723.

2. Kato N, Hirano T, Takeda K, Nakagawa N, Mizumoto T, Yuasa $\mathrm{H}$, et al. Treatment of aortic dissections with a percutaneous intravascular endoprosthesis: comparison of covered and bare stents. J Vasc Interv Radiol 1994;5:805-12.

3. Kato M, Matsuda T, Kaneko M, Ueda T, Kuratani T, Yoshioka Y, et al. Experimental assessment of newly devised transcatheter stent-graft for aortic dissection. Ann Thorac Surg 1995;59:90814.

4. Yoshida H, Yasuda K, Tanabe T. New approach to aortic dissection: development of an insertable aortic prosthesis. Ann Thorac Surg 1994;58:806-10.

5. Boudghène F, Sapoval M, Bigot JM, Michel JB. Endovascular graft placement in experimental dissection of the thoracic aorta. J Vasc Interv Radiol 1995;6:501-7.

6. Moon M, Dake MD, Pelc LR, Liddell R, Castro LJ, Mitchell RS, et al. Intravascular stenting of acute experimental type B dissections. J Surg Res 1993;54:381-8.

\section{Bound volumes available to subscribers}

Bound volumes of The Journal of Thoracic and Cardiovascular Surgery are available to subscribers (only) for the 1999 issues from the Publisher, at a cost of $\$ 134.00$ for domestic, $\$ 165.85$ for Canadian, and $\$ 155.00$ for international subscribers for Vol 117 (January-June) and Vol 118 (July-December). Shipping charges are included. Each bound volume contains a subject and author index and all advertising is removed. Copies are shipped within 60 days after publication of the last issue of the volume. The binding is durable buckram with the Journal name, volume number, and year stamped in gold on the spine. Payment must accompany all orders. Contact Mosby, Inc, Subscription Services, 11830 Westline Industrial Drive, St Louis, MO 63146-3318, USA; phone 800-453-4351 or 314-453-4351.

Subscriptions must be in force to qualify. Bound volumes are not available in place of a regular Journal subscription. 